\title{
La eficacia de la intervención asistida con delfines
}

\section{The effectiveness of assisted intervention with dolphins}

\section{Palabras clave}

Intervención asistida con delfines, educación especial, conceptos escolares fuera del aula, intervención asistida con animales.

\section{Keywords}

Assisted intervention with dolphins, special education, school concepts outside the classroom, assisted intervention with animals.

\section{Introducción}

La intervención asistida con animales es un programa educativo o terapéutico dirigido a alcanzar unos objetivos concretos con un usuario o un grupo de usuarios (Fine, 2003). Es necesario que sea evaluado y guiado por el profesional que ha marcado los objetivos a trabajar, según sea educación o terapia asistida con animales.

Se entiende por programa el conjunto de sesiones de intervención asistida con animales destinado a alcanzar unos objetivos concretos y definidos. Las premisas para realizar cualquier tipo de interacción con un animal deben estar especialmente entrenadas para ello, con una correcta socialización y unas habilidades específicas (Virnés y Buela, 2006). Actualmente, se realizan programas de intervención asistida con perros, caballos, animales de granja, leones marinos o delfines, entre otros. Cualquier programa de intervención requiere de la participación del entrenador del animal y del profesional o técnico que va a llevar a cabo las sesiones, anteponiendo siempre el bienestar tanto humano como animal.
Meritxell Arias Teixidor $<$ m.arias@discan.org>

Associació Discan

\section{Miguel Ángel Canseco González}

<mcanseco@delphinus.com.mx> Delphinus
Para citar:

Arias, M. y Canseco, M. A. (20I7):

"La eficacia de la intervención asistida con delfines". Revista Española de Discapacidad, 5 (2): 227-233.

Doi: <https://doi.org/I0.5569/23405I04.05.02.I4> 
Este proyecto se enmarca en una colaboración entre la empresa Delphinus y la Universitat de Barcelona, en el marco de la realización de las prácticas de los alumnos del Máster en Intervención Educativo-Terapéutica Asistida con Animales, y la Asociación de Acción Social Discan (en adelante Discan), con el objetivo de conocer los beneficios de la intervención asistida con delfines en niños/as con trastorno del espectro del autismo (TEA), parálisis cerebral (PC) y síndrome de Down (SD).

\section{Objetivos}

La intervención asistida con delfines tiene un ámbito de aplicación tan amplio que debemos especificar para cada uno de los participantes los objetivos a lograr. Sin embargo, diferenciamos claramente un objetivo general común a todos los participantes del programa de intervención:

- Aumentar la autonomía de cada uno de los usuarios mediante actividades destinadas a lograr objetivos específicos que permitan al usuario reducir el nivel de dependencia.

Teniendo en cuenta la sintomatología común de cada una de las discapacidades objeto de estudio, se plantearon dos objetivos concretos para cada una de ellas:

\section{Trastorno del espectro del autismo}

I. Aumentar el contacto visual.

2. Reducir la conducta estereotipada.

Mediante el logro de estos dos objetivos ofrecemos al usuario una mejora en la comunicación, por lo que la dependencia que tiene al no poder comunicarse se verá reducida.

\section{Síndrome de Down}

I. Mejorar la conducta.

2. Respetar las órdenes y normas.

Alcanzando una mejora de la conducta, ofrecemos al usuario un conocimiento del saber estar en las situaciones cotidianas, con lo que le dotamos de autonomía social.

\section{Parálisis cerebral}

I. Mejorar el control de tronco nivel cefálico y torácico.

2. Mejorar la funcionalidad de las extremidades superiores

Es evidente que aumentar el nivel de funcionalidad de las extremidades superiores y el control de tronco y cabeza ofrece al usuario una menor dependencia para dirigir sus movimientos donde él requiera en cada momento.

Estos objetivos fueron propuestos por diversas razones. En primer lugar, los objetivos propuestos para el grupo de niños/as con trastorno del espectro del autismo fueron planteados teniendo en cuenta información que Dave Nathanson presentó en el 'Congress Proceedings of the XVI World Assembly of the World Organization for Preschool Education' en I980, por la que un niño no verbal tenía cuatro veces más probabilidades de responder antes a un delfín que a su madre (Nathanson, I980).

Teniendo en cuenta que el primer paso para establecer comunicación verbal es el contacto visual, uno de nuestros objetivos para los niños/as sin éste tenía que ser aumentarlo y probar que la interacción con el delfín potenciaría ese interés.

En el caso de los niños/as con síndrome de Down, escogimos los dos objetivos propuestos ayudándonos del conocimiento que se desprende de una segunda investigación de Dave Nathanson, en este caso junto a De Faria (Nathanson y De Faria, I993), por la que niños de ocho años con síndrome de Down respondieron mejor usando interacciones con delfines como reforzadores que usando interacciones con sus juguetes preferidos. También tuvimos en cuenta en este caso el estudio de Eloy J. Pineda por el que el 83,3\% de los padres de los participantes refirieron un mayor avance en el aprendizaje (Pineda, 2008).

En los niños/as con parálisis cerebral los dos objetivos fueron elegidos teniendo en cuenta 
que el agua es, por lo general, un medio idóneo y adecuado para el desarrollo y consecución de un gran número de objetivos en las áreas de desarrollo físico y psicomotor. Como elemento que se adapta a las características específicas de la persona, el agua es ideal para planteamientos de programas y trabajos personalizados (Villagra, 2007).

\section{Metodología}

El presente proyecto se desarrolló en las instalaciones de uno de los delfinarios de la empresa GVD en la Riviera Maya, específicamente 'Delphinus Riviera Maya', durante el mes de septiembre de 20I3. Se basó en la realización de un programa de intervención asistida con delfines en doce niños(as) con diferentes discapacidades, a razón de tres niños por día: uno con SD, uno con TEA y el tercero con PC, a quienes denominamos usuarios, y a partir de una metodología basada en conceptos escolares y un animal como elemento motivador. Los usuarios seleccionados presentaron autorización médica así como familiar y, por otra parte, su autorización para el uso y publicación de las imágenes y grabaciones derivadas de este trabajo. Los doce usuarios fueron seleccionados por la directora de la asociación Discan, así como por personal del Sistema Nacional para el Desarrollo Integral de la Familia (DIF) de Quintana Roo, y en colaboración con sus terapeutas se realizaron las valoraciones iniciales de cada uno de ellos.

Para poder alcanzar los objetivos propuestos fue necesario marcar criterios de inclusión y exclusión, así como características específicas de cada tipo de discapacidad. En ese sentido, los criterios de inclusión comunes fueron: a) niños de cuatro a diez años, b) niños diagnosticados, c) niños con sintomatología clara y d) interés y compromiso familiar por participar.

Por otra parte, los criterios de exclusión comunes quedaron como sigue: a) epilepsia no controlada (puede ser medicada pero no controlada), b) miedo al agua y c) inestabilidad atlas-axis complicada (únicamente para los casos de SD).

Se propusieron las siguientes características relativas a los usuarios con TEA: I) autismo puro (no síndrome de asperger o de rett), 2) conducta estereotipada, 3 ) ausencia de contacto visual y 4) ausencia de conductas espontáneas para compartir juguetes o intereses.

Respecto a los usuarios con SD fueron las siguientes: I) comportamiento impulsivo, 2) deficiencia en la capacidad de discernimiento y 3) falta de autonomía.

Las características planteadas para los usuarios con PC fueron: I) parálisis cerebral tipo espástica, 2) preferiblemente diplejía espástica, pero puede ser cuadriplejia y 3) puede tener o no déficit en la percepción espacial.

Las sesiones se realizaron de lunes a sábado dividiéndose en tres partes. La primera de climatización al medio acuático y previo al contacto con el delfín, la segunda de contacto con el delfín y la tercera de trabajo fuera del agua con el técnico de intervención asistida con animales. Todas las sesiones de agua fueron acompañadas por sesiones en tierra, en las que los usuarios pudieron recordar y repetir las actividades trabajadas en el agua.

En la intervención asistida con animales, se utiliza al animal como elemento motivador para alcanzar unos objetivos concretos más rápidamente. El proyecto se realizó adaptando actividades de aula a la presencia de un delfín y a su realización en el exterior y en agua. Para ello, los materiales se adaptaron para su uso en el agua, siendo de gran tamaño para facilitar su manipulación con el uso de las dos manos. Asimismo, se creó nuevo material de gran tamaño y de colores vivos para focalizar la atención de los usuarios.

Las actividades principales realizadas fueron las siguientes, cada una en una sesión distinta: 
- Actividad I: actividad destinada a conocer las características principales del delfín mediante la realización de preguntasrespuestas. El usuario lanza un dado cuyas caras en vez de números tienen colores los que, a su vez, estarán vinculados a unas preguntas. Así, el usuario debe ir a buscar la pregunta que tenga el mismo el color que le haya tocado. Cada pregunta tiene dos opciones de respuesta en modo de fotografía. Una vez el usuario da la respuesta, se le enseña al delfín la tarjeta con la fotografía seleccionada y asentirá o negará en función de si es o no la correcta. Después se llevará a cabo la actividad que haya propuesta en la parte trasera de la tarjeta.

- Actividad 2: el usuario lanza un dado cuyos colores estarán relacionados con unos plafones cuya información revelará con qué parte del cuerpo debe el niño tocar cierta parte del delfín. Ejemplo: Azul $\rightarrow$ Mano Derecha $\rightarrow$ Aleta Dorsal.

- Actividad 3: el usuario lanza el dado de colores, que esta vez están relacionados con una actividad que deberá realizar con el delfín. Ejemplo: Rojo $\rightarrow$ El usuario debe lanzarle una pelota al delfín y este se la devuelve.

- Actividad 4: el usuario debe armar un aro a partir de la unión de nueve piezas que están agrupadas de tres en tres en función de su color (hay tres verdes, tres rojos y tres azules). De cada grupo se dará al participante dos de las tres partes para que las junte y la última se la lanzará al delfín, que se encargará de devolverlo para poder acabar de unir todas las piezas del mismo color. Esto se repite tres veces (tres colores) y, una vez tenemos todas las partes, las unimos para formar un aro grande. Finalmente, le pedimos al usuario que pase por el aro para mostrarle al delfín "cómo se debe hacer" y pueda terminar el ejercicio pasando por él mientras el usuario y el terapeuta agarran el aro uno por cada lado.

- Actividad 5: en el agua hay materiales de tres colores (rojo, verde y naranja) que el usuario deberá recoger y colocar en un recipiente. La clasificación dependerá de los colores de los materiales.

Concretamente hay tres recipientes, uno de color naranja, otro verde y otro rojo y cada uno de ellos contiene una fotografía con la actividad que deben hacer el usuario y el delfín, como nadar juntos, hacerlo cantar o jugar a pelota.

- Actividad 6: antes de iniciar la actividad hacemos un recordatorio de las actividades realizadas durante la semana. Pondremos las tarjetas con cada actividad en un plafón de manera ordenada, para realizarlas de forma progresiva a modo de finalización de programa, buscando contacto directo con el delfín entre actividad y actividad para agradecerle todo lo realizado.

Durante las sesiones, el técnico ayudó y motivó verbalmente en todo momento, intentando que el usuario no se distrajera con el entorno. Además, la distribución de las actividades se realizó marcando unas rutinas muy concretas.

Es bien sabido que las rutinas en educación constituyen situaciones de aprendizaje que los niños y niñas realizan diariamente, de forma estable y permanente. En la educación especial es básico respetar los ritmos del desarrollo cognitivo y socioafectivo a nivel individual, manteniendo una organización del tiempo lo más natural posible, sin forzar el ritmo de la actividad y manteniendo determinadas constantes temporales.

Estas constantes temporales están marcadas por las rutinas diarias. Los niños necesitan que estas rutinas tengan siempre la misma secuencia para sentirse seguros y alcanzar grandes cotas de autonomía y equilibrio personal (Moratalla y Martínez, 2009).

No obstante, las rutinas no son actividades rígidas y mecánicas, sino que permiten al niño anticipar acontecimientos, relacionar tiempos y espacios (Figura I).

Teniendo esto en cuenta, las sesiones estuvieron estructuradas, por una parte, desde el punto 


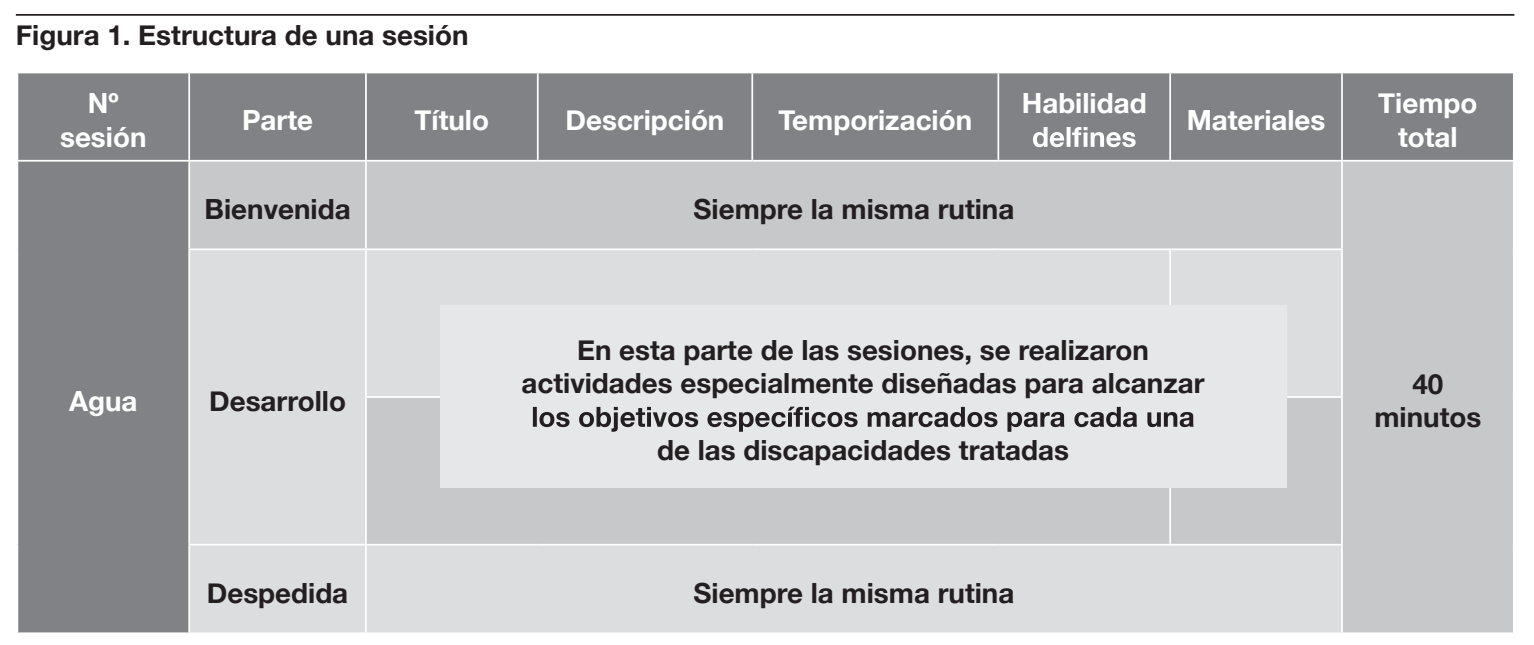

Fuente: elaboración propia.

de vista del proceso de enseñanza-aprendizaje, donde las actividades de bienvenida y despedida se convirtieron en el elemento organizador de las demás actividades y, por otra, desde el punto de vista del desarrollo personal, donde las actividades marcaron situaciones de interacción que contribuyeron al establecimiento de relaciones personales del niño con sus iguales, permitiéndole su inserción social, que se traduce en la capacidad de sentirse miembro de un grupo y de poder actuar de forma cada vez más autónoma en él.

\section{Resultados}

En los usuarios con TEA, para el primer objetivo (aumentar el contacto visual), se contó el número de veces que se les pidió que miraran y el número de veces que miraron. Para el segundo objetivo (reducir la conducta estereotipada), se contabilizó el número de estereotipias de cada sesión en uno de los usuarios dado que los demás sí mostraron conducta estereotipada fuera del agua pero no dentro, por lo que podríamos decir que en las sesiones mejora un roo \% su conducta estereotipada.
Se midieron sólo tres de los cuatro usuarios participantes del programa, dado que uno de ellos mostró hipersensibilidad al agua y se tuvieron que realizar las sesiones desde fuera. En los tres casos pudimos corroborar un aumento del contacto visual a lo largo del programa. En el caso de los usuarios con SD, se tuvieron en cuenta los cuatro participantes del programa. Se plantearon dos objetivos: mejorar la conducta (impulsividad, oposición y escape) y respetar las normas.

Los cuatro usuarios con síndrome de Down mejoraron en sus objetivos aumentando sus respuestas aproximadamente en un $20 \%$ en cada uno de los ítems establecidos. No todos los usuarios necesitaron ser valorados en los tres ítems del objetivo "Mejorar la conducta", ya que cada uno de ellos mostró una conducta disruptiva distinta.

En el caso de los usuarios con parálisis cerebral, se tuvieron en cuenta los dos participantes que participaron en el programa. En este caso, se mantuvieron los objetivos iniciales: mejorar el control de tronco (cefálico o torácico, según las capacidades del usuario) y mejorar la funcionalidad de las extremidades superiores. Una vez analizados los resultados de este último grupo no se ha podido constatar ninguna mejoría dado que la temperatura del agua, al 
ser inferior a $28^{\circ}$, no ha ayudado a mejorar la espasticidad de los usuarios (para que se dé una reducción es necesario que el agua esté a mínimo $\left.30^{\circ}\right)$. Este hecho, junto con el clima del momento (muchos días fueron lluviosos) han llevado a pensar que con este tipo de usuarios se debe trabajar en épocas más calurosas y hacerlo siempre en una posición corporal estable, ya que el oleaje entorpece la mejora de la postura corporal para realizar los ejercicios de la mejor manera posible y poder alcanzar los objetivos establecidos.

Teniendo en cuenta los condicionantes, $\mathrm{y}$ sabiendo que la espasticidad influye al Ioo \% en el control postural y de movimientos, creemos que los objetivos no han sido alcanzados por no haber conseguido rebajar la espasticidad, y no por la realización de la intervención propiamente dicha.

\section{Conclusiones}

La intervención asistida con animales es beneficiosa para los participantes de la misma en muchos aspectos, tanto físicos como psicológicos y emocionales.

En el caso de la intervención asistida con delfines, hemos podido constatar que todos los participantes del programa han mejorado en cada uno de los objetivos tratados, sin tener en cuenta los usuarios con parálisis cerebral, con los que deberíamos trabajar en las épocas menos lluviosas, y en las horas en las que el agua esté a la más alta temperatura, ya que ello nos ayudará a realizar movimientos con usuarios con espasticidad elevada.

Teniendo en cuenta que todos los usuarios han mejorado en sus objetivos, es conveniente pensar que la intervención asistida con delfines es beneficiosa. Seguramente deberíamos seguir trabajando para conocer si existen patrones específicos para los diferentes grupos de discapacidad y en qué frecuencia sería mejor realizar las sesiones de intervención asistida con delfines.

Es necesario que los usuarios conozcan antes de empezar a los técnicos que participaran en el programa, creemos que este ha sido un hándicap en el proyecto, ya que el trato hacia los técnicos ha sido distinto según ha ido aumentando la confianza con los mismos y esto puede influir en los resultados.

También es necesario tener en cuenta el conjunto de actividades que se realizan simultáneamente con las sesiones, dado que ha habido situaciones en las que ha habido mucho ruido alrededor y esto influye directamente en la atención de los participantes, por lo que en futuras evaluaciones se deberán contar como variables. 


\section{Referencias bibliográficas}

Fine, A. H. (2003): Manual de Terapia Asistida con Animales: fundamentos teóricos y modelos prácticos. Fundación Affinity.

Moratalla, I. S. y Abel, M. G. (2009): Manual del usuario. Rutinas. Colegio de Educación Especial Eloy Camino.

Nathanson, D. E. (I980): "Dolphin and kids: A communication experiment". En: Congress Proceedings of the XVI World Assembly of the World Organization for Preschool Education.

Nathanson, D. E. y De Faria, S. (I993): "Cognitive improvement of children in water with and without dolphins” Anthrozoös, 6 (I), I7-29.
Pineda Pérez, E. J. (2008): “La delfinoterapia en niños con síndrome Down atendidos en un círculo infantil especial”. Revista Cubana Pediátrica, 80 (4): ०-०.

Villagra, A. H. A. (2007): "Hidroterapia y actividad acuática adaptada: Parálisis Cerebral, Autismo, Síndrome de Down”. Instituto Andaluz del Deporte.

Virnés-Ortega, J. y Gualberto, B. C. (2006): "Psycho physiological Effects of HumanAnimal Interaction: Theoretical Issues and Long-Term Interaction Effects”. Journal of Nervous and Mental Disease, I94 (I): 52-57. 